\title{
Valve Dysfunction of Injection Port Causing the Leaking Intravenous Cannula
}

\author{
Amarjeet Kumar ${ }^{1}$, Neeraj Kumar ${ }^{2}$, Abhyuday Kumar ${ }^{3}$, Ajeet Kumar ${ }^{4}$, Sanjeev Kumar ${ }^{5}$
}

\begin{abstract}
We report a situation of leaking peripheral intravenous cannula (PIVC) due to dysfunction of the injection port valve. After shifting the patient to the operating room, anesthesia monitors were attached. The patient was having a 20-G peripheral intravenous cannula (Vygonule V, Vygon Haryana India Pvt. Ltd.) in situ over the dorsum of the right hand. We noticed that fluid is coming out freely through the injection port (Fig. 1). The PIVC was removed and the puncture site was properly dressed. Another PIVC was secured over the dorsum of the left hand. However, we did not have a suitable guidewire to replace it and had to place it afresh. Also, using such a set to replace the PIVC would involve significant costs which might be more than the cost of the cannula. The quality control unit of the manufacturing company should be notified of such an incident as was done in our case.
\end{abstract}

Keywords: Guidewire, Hypovolemic shock, Peripheral intravenous cannula.

Panamerican Journal of Trauma, Critical Care \& Emergency Surgery (2021): 10.5005/jp-journals-10030-1304

We report a situation of leaking peripheral intravenous cannula (PIVC) due to dysfunction of the injection port valve. After shifting the patient to the operating room, anesthesia monitors were attached. The patient was having a $20-\mathrm{G}$ peripheral intravenous cannula (Vygonule V, Vygon Haryana India Pvt. Ltd.) in situ over the dorsum of the right hand. An intravenous (IV) set with Ringer's lactate was then attached and the roller flow controller was opened. As fluid was not going through the IV set, the cannula was flushed with $2 \mathrm{~mL}$ of normal saline through the injection port. We noticed that fluid is coming out freely through the injection port (Fig. 1). The PIVC was removed and the puncture site was properly dressed. Another PIVC was secured over the dorsum of the left hand. Up to $40 \%$ of peripheral PIVCs may be complicated by kinking, blockage, dislodgement, or infection. ${ }^{1}$ However, the leaking injection port of a PIVC is an infrequent complication necessitating its replacement.

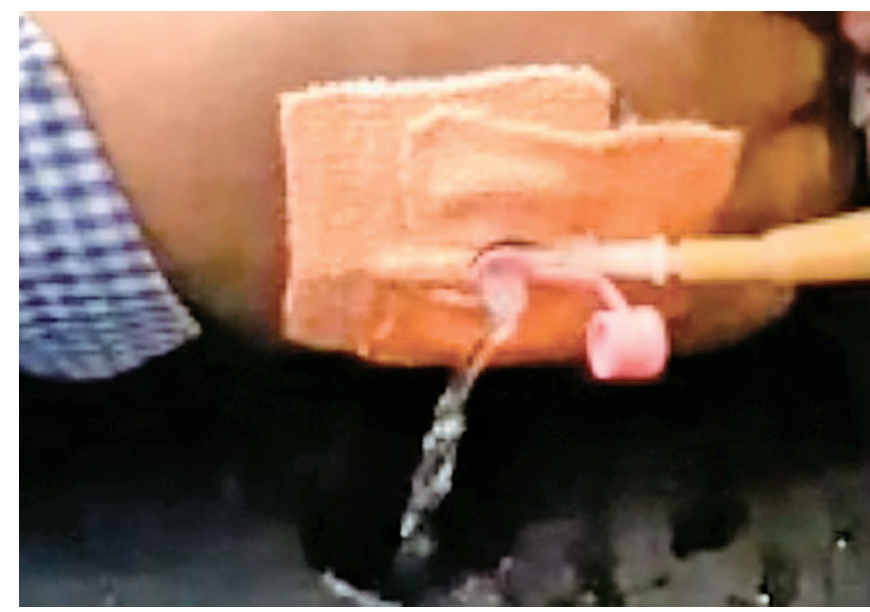

Fig. 1: Leaking intravenous cannula through an injection port
1,2Department of Trauma and Emergency, All India Institute of Medical Sciences, Patna, Bihar, India

3,4 Department of Anaesthesiology, All India Institute of Medical Sciences, Patna, Bihar, India

${ }^{5}$ Department of CTVS, All India Institute of Medical Sciences, Patna, Bihar, India

Corresponding Author: Neeraj Kumar, Department of Trauma and Emergency, All India Institute of Medical Sciences, Patna, Bihar, India, Phone: +91 8210104972, e-mail: neeraj.jInmc@gmail.com

How to cite this article: Kumar A, Kumar N, Kumar A, et al. Valve Dysfunction of Injection Port Causing the Leaking Intravenous Cannula. Panam J Trauma Crit Care Emerg Surg 2021;10(1):56-57.

Source of support: Nil

Conflict of interest: None

Cannula failure and its replacement is often time-consuming and painful, especially in patients with difficult access and younger age.

Very few cases of leaking PIVC are reported due to the dysfunction of injection port valves in the literature. ${ }^{2}$ Many a time securing a PIVC can often be difficult and time-consuming and if not inserted promptly may result in hypovolemia. The cause of the leaking PIVC in our case was presumably due to damage of the one-way valve present inside the injection port during the flushing of the IV cannula. Repeated withdrawal and reinsertion of the needle inside the cannula may damage the valve (elastomeric sleeve) and is not recommended by the manufacturer. ${ }^{3}$ Another option for the management of this complication can be the replacement of the leaking PIVC over a guidewire, especially in difficult situations. A report of an 18-month-old Holt-Oram syndrome child described the use of a 23-G arterial catheter (LeaderCath ${ }^{\oplus}$ ) guidewire to exchange a leaking PIVC with a fresh one. ${ }^{4}$ In that case, the cannula was leaking at the junction of the shaft with the hub. However, we did not have a suitable guidewire to replace it and had to place

\footnotetext{
(c) The Author(s). 2021 Open Access This article is distributed under the terms of the Creative Commons Attribution 4.0 International License (https:// creativecommons.org/licenses/by-nc/4.0/), which permits unrestricted use, distribution, and non-commercial reproduction in any medium, provided you give appropriate credit to the original author(s) and the source, provide a link to the Creative Commons license, and indicate if changes were made. The Creative Commons Public Domain Dedication waiver (http://creativecommons.org/publicdomain/zero/1.0/) applies to the data made available in this article, unless otherwise stated.
} 
it afresh. Also, using such a set to replace the PIVC would involve significant costs which might be more than the cost of the cannula. The quality control unit of the manufacturing company should be notified of such an incident as was done in our case.

\section{References}

1. Wallis MC, McGrail M, Webster J, et al. Risk factors for peripheral intravenous catheter failure: a multivariate analysis of data from a randomized controlled trial. Infect Control Hosp Epidemiol 2014;35:63-68. DOI: 10.1086/674398.

2. Behura A, Ahuja M. A leaky intravenous cannula. Anaesthesia 2006;61:411. DOI: 10.1111/j.1365-2044.2006.04603.x.

3. Dwivedi P, Kumar A. Injection port valve malfunction!. Egypt J Anaesth 2017;33(4):385-386. DOI: 10.1016/j.egja.2017.07.004.

4. Singh P, Kishore K. Guidewire replacement of leaking paediatric intravenous cannula. Indian J Anaesth 2016;60:70-71. DOI: 10.4103/0019-5049.174814. 